\title{
Traditional japanese (kampo) medicine yokukansan for behavioral and psychiatric symptoms of dementia in alzheimer's disease
}

Volume 5 Issue 5 - 2017

\section{Opinion}

Behavioral and psychiatric symptoms of dementia (BPSD) are among the most troubling symptoms in Alzheimer's disease (AD) patients. In 2005 and 2008, the Food Drug Administration reported that "antipsychotics are not indicated for the treatment of dementiarelated psychosis." Yet, the available therapeutic options for AD patients with BPSD are few. ${ }^{1}$

In Japan, Kampo medicine provides clinicians and patients an alternative therapeutic option when modern medicine is ineffective. Kampo medicine Yokukansan (YKS) is gaining popularity as a clinical treatment for BPSD in Japan. YKS is typically used in conjunction with acetylcholinesterase inhibitors or NMDA receptor antagonists, which are used to treat the core symptoms of $\mathrm{AD} .^{2}$

Although most forms of Kampo medicine lack sufficient evidence, YKS is an exception, and multiple clinical studies have been performed. Previous clinical studies of YKS have reported its efficacy as a BPSD treatment. Iwasaki et al. found that several BPSD symptoms, including aggression, agitation, and irritability, improved with YKS in patients with mild to severe dementia including AD. ${ }^{3}$ In addition, the efficacy of YKS was reported in a systematic review and meta-analysis. $^{4}$

Studies of YKS are underway not only in clinical settings but also in fundamental pharmacology. Reportedly, transport of glutamate, gamma-aminobutyric acid (GABA), and serotonin may be associated with BPSD. ${ }^{5}$ Several pharmacologic mechanisms of action have been recently described for YKS, including improved glutamate uptake, inhibition of glutamate-induced neuronal death, modulation of GABA receptor expression, and partial agonist effect on serotonin 5-HT 1A receptors. ${ }^{6-8}$ The Japan Geriatrics Society Working Group recommend the use of YKS in AD patients with BPSD. ${ }^{9} 10$ YKS uses Glycyrrhizae radix (licorice root); therefore, hypokalemia, hypertension, and edema are potential adverse effects and require monitoring.

\section{Acknowledgments}

None.

\section{Conflicts of interest}

Author declares there are no conflicts of interest.

\section{Funding}

None.

\section{References}

1. h t t p : // w w w. fda.gov/d r ug s/d rug s a fe ty/ postmarketdrugsafetyinformationforpatientsandproviders/ucm 124830 . html

\author{
Takehiro Numata , Shin Takayama \\ Department of Kampo medicine, Tohoku University Hospital, \\ Japan
}

Correspondence: Shin Takayama, Associate professor, Department of Kampo medicine, Tohoku University Hospital, I-I, Seiryo-machi, Aobaku, Sendai city, Miyagi prefecture, Japan, Tel 8I-22-7I7-7587, Email takayama@med.tohoku.jp

Received: February 14, 2017 | Published: February 21, 2017

2. Howes MJR, Perry E. The role of phytochemicals in the treatment and prevention of dementia. Drugs and Aging. 2011;28(6):439-468.

3. Iwasaki K, Satoh-Nakagawa T, Maruyama M, et al. A randomized, observer-blind, controlled trial of the traditional Chinese medicine YiGan San for improvement of behavioral and psychological symptoms and activities of daily living in dementia patients. $J$ Clin Psychiatry. 2005;66(2):248-252.

4. Matsuda Y, Kishi T, Shibayama H, et al. Yokukansan in the treatment of behavioral and psychological symptoms of dementia: a systematic review and meta-analysis of randomized controlled trials. Hum Psychopharmacol. 2013;28(1):80-86.

5. Steele C1, Rovner B, Chase GA,et al. Psychiatric symptoms and nursing home placement of patients with Alzheimer's disease. Am J Psychiatry. 1990;147(8):1049-1051.

6. Kawakami Z, Kanno H, Ueki T, et al. Neuroprotective effects of Yokukansan, a traditional Japanese medicine, on glutamate-mediated excitotoxicity in cultured cells. Neuroscience. 2009;159(4):1397-1407.

7. Egashira N, Nogami A, Iwasaki K, et al. Yokukansan enhances pentobarbital-induced sleep in socially isolated mice: possible involvement of GABA (A)-benzodiazepine receptor complex. $J$ Pharmacol Sci. 2011;116(3):316-320.

8. Meltzer HY, Sumiyoshi T. Does stimulation of 5-HT (1A) receptors improve cognition in schizophrenia? Behav Brain Res. 2008;195(1):98102 .

9. Kojima T, Mizukami K, Tomita N, et al. Screening Tool for Older Persons' Appropriate Prescriptions in Japanese: Report of the Japan Geriatrics Society Working Group on "Guidelines for medical treatment and its safety in the elderly". Geriatr Gerontol Int. 2016;16(9):983-1001.

10. Takayama S, Iwasaki K. Systematic review of traditional Chinese medicine for geriatrics. Geriatr Gerontol Int. 2016;10.1111.12803. 